

\title{
Extremality of loci of hyperelliptic curves with marked Weierstrass points
}

\author{
Dawei Chen and Nicola Tarasca
}

\begin{abstract}
The locus of genus-two curves with $n$ marked Weierstrass points has codimension $n$ inside the moduli space of genus-two curves with $n$ marked points, for $n \leq 6$. It is well known that the class of the closure of the divisor obtained for $n=1$ spans an extremal ray of the cone of effective divisor classes. We generalize this result for all $n$ : we show that the class of the closure of the locus of genus-two curves with $n$ marked Weierstrass points spans an extremal ray of the cone of effective classes of codimension $n$, for $n \leq 6$. A related construction produces extremal nef curve classes in moduli spaces of pointed elliptic curves.
\end{abstract}

Every smooth curve of genus two has a unique map of degree two to the projective line, ramified at six points, called Weierstrass points. It follows that the locus $\mathcal{H} y p_{2, n}$ of curves of genus two with $n$ marked Weierstrass points has codimension $n$ inside the moduli space $\mathcal{M}_{2, n}$ of smooth curves of genus two with $n$ marked points, for $1 \leq n \leq 6$. In this paper, we study the classes of the closures of the loci Hyp $p_{2, n}$ inside the moduli space of stable curves $\overline{\mathcal{M}}_{2, n}$.

The cone of effective codimension-one classes on $\overline{\mathcal{M}}_{2,1}$ is explicitly described in [Rulla 2001; 2006], and encodes the rational contractions of $\overline{\mathcal{M}}_{2,1}$. It is thus natural to study cones of effective classes of higher codimension. The following is one of the first results in this direction.

Theorem 1. For $1 \leq n \leq 6$, the class of $\overline{\mathcal{H} y p}_{2, n}$ is rigid and extremal in the cone of effective classes of codimension $n$ in $\overline{\mathcal{M}}_{2, n}$.

Theorem 1 motivates the computation of the classes of the loci $\overline{\mathcal{H} y p}_{2, n}$. The class of the divisor $\overline{\mathcal{H} y p}_{2,1}$ has been computed in [Eisenbud and Harris 1987], and the class of the codimension-two locus $\overline{\mathcal{H} y p}_{2,2}$ has been computed in [Tarasca 2015]. In Section 5 we study the next nontrivial case.

During the preparation of this article, Chen was partially supported by NSF CAREER grant DMS1350396.

MSC2010: primary 14H99; secondary 14C99.

Keywords: subvarieties of moduli spaces of curves, effective cones, higher codimensional cycles. 
Theorem 2. In $A^{3}\left(\overline{\mathcal{M}}_{2,3}\right)$, we have

$$
\begin{aligned}
& {\left[\overline{\mathcal{H} y p}_{2,3}\right]=\left(\left(3 \omega_{1}-\lambda-\delta_{1}\right) \cdot\left(3 \omega_{2}-\lambda-\delta_{1}\right)\right.} \\
&\left.-\left(\delta_{0:\{1,2\}}+\delta_{0: 3}\right) \cdot\left(3 \omega_{1}-\lambda-\delta_{1}\right)-\gamma_{1: \varnothing}-\gamma_{1:\{3\}}\right) \\
& \cdot\left(3 \omega_{3}-\lambda-\delta_{1}-\delta_{0:\{1,3\}}-\delta_{0:\{2,3\}}\right) \\
&-\gamma_{1:\{1\}} \cdot\left(2 \psi_{1}-\delta_{1:\{1\}}\right)-\gamma_{1:\{2\}} \cdot\left(2 \psi_{2}-\delta_{1:\{2\}}\right)-\gamma_{1: \varnothing} \cdot\left(\psi_{1}-\delta_{0:\{1,3\}}\right) .
\end{aligned}
$$

For elliptic curves, the difference of two ramification points of a degree- 2 map to the projective line can be regarded as a 2-torsion point. In a somewhat similar fashion, we consider in general the locus of points on elliptic curves whose pairwise differences are $k$-torsion points. More precisely, for $k \geq 2$ and $2 \leq n \leq k^{2}$, consider the following one-dimensional locus in $\mathcal{M}_{1, n}$ :

$$
\mathcal{T o r}_{1, n}^{k}:=\left\{\left[C, p_{1}, \ldots, p_{n}\right] \in \mathcal{M}_{1, n} \mid k p_{1} \sim \cdots \sim k p_{n}\right\} .
$$

Note that $\mathcal{T o r}_{1, n}^{k}$ might be reducible: $\mathcal{T o r}_{1, n}^{d}$ is a subcurve of $\mathcal{T} r_{1, n}^{k}$ for all divisors $d$ of $k$.

The class of the divisor $\overline{\mathcal{T o r}}_{1,2}^{2}$ is in the interior of the two-dimensional cone of effective divisor classes in $\overline{\mathcal{M}}_{1,2}$ and spans an extremal ray of the cone of nef divisor classes in $\overline{\mathcal{M}}_{1,2}$ [Rulla 2001].

Theorem 3. For $k \geq 2$ and $2 \leq n \leq k^{2}$, the class of $\overline{\mathcal{T o r}}_{1, n}^{k}$ spans an extremal ray of the cone of nef curve classes in $\overline{\mathcal{M}}_{1, n}$, and this ray does not dependent on $k$.

Structure of the paper. The proof of Theorem 3 is in Section 1 - this section is independent from the rest of the paper. In Section 2 we collect some classical results on classes of hyperelliptic loci which are needed later on. The proof of Theorem 1 in the case $n=2$ is in Section 4.2 and is based on the explicit description of the codimension-two class $\left[\overline{\mathcal{H} y p}_{2,2}\right]$ presented in Section 4.1. In Section 3 we prove a recursive argument that works in a more general context, and we thus complete the proof of Theorem 1. Finally, we prove Theorem 2 in Section 5 using the description of the classes $\left[\overline{\mathcal{H} y p}_{2,1}\right]$ and $\left[\overline{\mathcal{H} y p}_{2,2}\right]$ from Section 4.1 .

Notation. We use throughout the following notation for divisor classes on $\overline{\mathcal{M}}_{g, n}$. The class $\psi_{i}$ is the cotangent class at the marked point $i$, and the class $\omega_{i}$ is the pullback of the class $\psi_{i}$ via the map $\rho_{i}: \overline{\mathcal{M}}_{g, n} \rightarrow \overline{\mathcal{M}}_{g, 1}$ obtained by forgetting all marked points but the point $i$. The class $\delta_{\text {irr }}$ is the class of the closure of the locus of nodal irreducible curves. We denote by $\lambda$ the pullback of the first Chern class of the Hodge bundle over $\overline{\mathcal{M}}_{g}$. For $i \in\{0, \ldots, g\}$ and $J \subseteq\{1, \ldots, n\}$, we denote by $\delta_{i: J}$ the class of the divisor $\Delta_{i: J}$ whose general element has a component of genus $i$ containing the points marked by indices in $J$ and meeting a component of genus $g-i$ containing the remaining marked points. One has $\delta_{i: J}=\delta_{g-i: J^{c}}$. We denote by $\delta_{i: j}$ the sum of all distinct divisor classes $\delta_{i: J}$ such that $|J|=j$, and by $\delta_{i}$ the 
sum of all distinct classes $\delta_{i: j}$ for all possible values of $j$. Let $\pi_{k}: \overline{\mathcal{M}}_{g, n} \rightarrow \overline{\mathcal{M}}_{g, n-1}$ be the map obtained by forgetting the $k$-th marked point. Note that $\pi_{k}^{*}\left(\delta_{i}\right)=\delta_{i}$ on $\overline{\mathcal{M}}_{g, n}$ for $n \geq 2$.

We also use the following codimension-two tautological classes on $\overline{\mathcal{M}}_{g, n}$. For $J \subseteq\{1, \ldots, n\}$, let $\gamma_{1: J}$ be the class of the locus $\Gamma_{1: J}$ of curves whose general element has an elliptic component containing exactly the points marked by indices in $J$, and meeting in two points a component of genus $g-2$ containing the remaining marked points.

Throughout we work over an algebraically closed field of characteristic 0 . All cycle classes are stack fundamental classes, and all cohomology and Chow groups are taken with rational coefficients. We implicitly assume real coefficients when we consider nef classes and closures of cones of effective classes.

\section{Extremal nef curve classes on $\overline{\mathcal{M}}_{1, n}$}

In this section we show that the class of the one-dimensional locus $\overline{\mathcal{T}}_{1, n}^{k}$ spans an extremal ray of the cone $\mathrm{Nef}^{n-1}\left(\overline{\mathcal{M}}_{1, n}\right)$ of nef curve classes on $\overline{\mathcal{M}}_{1, n}$, for $k \geq 2$ and $2 \leq n \leq k^{2}$.

By definition, the cone $\operatorname{Nef}^{n-1}\left(\overline{\mathcal{M}}_{1, n}\right)$ is dual to the cone of pseudoeffective divisors $\overline{\operatorname{Eff}}^{1}\left(\overline{\mathcal{M}}_{1, n}\right)$. A subcone $S$ of $\overline{\operatorname{Eff}}^{1}\left(\overline{\mathcal{M}}_{1, n}\right)$ is extremal in $\overline{\operatorname{Eff}}^{1}\left(\overline{\mathcal{M}}_{1, n}\right)$ if whenever $E_{1}+E_{2} \in S$, then $E_{1}, E_{2} \in S$. We first show that the cone generated by the boundary divisor classes is extremal in $\overline{\operatorname{Eff}}^{1}\left(\overline{\mathcal{M}}_{1, n}\right)$.

Lemma 4. The cone generated by the classes $\delta_{0: J}$ for $J \subseteq\{1, \ldots, n\}$ is extremal in $\overline{\operatorname{Eff}}^{1}\left(\overline{\mathcal{M}}_{1, n}\right)$ for $n \geq 2$.

Proof. We follow the strategy of [Rulla 2001, Corollary 1.4.7], where Rulla shows that the cone generated by the boundary divisor classes on $\overline{\mathcal{M}}_{g}$ is extremal in $\overline{\operatorname{Eff}}^{1}\left(\overline{\mathcal{M}}_{g}\right)$.

We first show that the classes $\delta_{0:\{i, j\}}$ are extremal in $\overline{\operatorname{Eff}}^{1}\left(\overline{\mathcal{M}}_{1, n}\right)$, for $n \geq 3$ and $i, j \in\{1, \ldots, n\}$. Consider the one-dimensional family of curves $C_{\{i, j\}}$ obtained by attaching a rational component containing the points with markings $i$ and $j$ at a moving point of an elliptic curve containing the remaining $n-2$ marked points. The curve $C_{\{i, j\}}$ is a moving curve in $\Delta_{0:\{i, j\}}$, and one has $C_{\{i, j\}} \cdot \delta_{0:\{i, j\}}<0$. It follows that $\delta_{0:\{i, j\}}$ is rigid and extremal in $\overline{\operatorname{Eff}}^{1}\left(\overline{\mathcal{M}}_{1, n}\right)$. Moreover, $C_{\{i, j\}}$ has empty intersection with $\delta_{0: J}$ for $|J|=2$ and $J \neq\{i, j\}$. From [Rulla 2001, Lemma 1.4.6], the cone generated by the classes $\delta_{0: J}$ for $|J|=2$ is extremal in $\overline{\operatorname{Eff}}^{1}\left(\overline{\mathcal{M}}_{1, n}\right)$.

We then use the following recursion on $k$, for $3 \leq k \leq n-1$. Suppose that the cone generated by all classes $\delta_{0: J}$ with $|J|<k<n$ is extremal in $\overline{\operatorname{Eff}}^{1}\left(\overline{\mathcal{M}}_{1, n}\right)$. For $i_{1}, \ldots, i_{k} \in\{1, \ldots, n\}$, consider the one-dimensional family $C_{\left\{i_{1}, \ldots, i_{k}\right\}}$ obtained by attaching a rational component containing the points with markings $i_{1}, \ldots, i_{k}$ at a moving point of an elliptic curve containing the remaining $n-k$ marked points. One 
has $C_{\left\{i_{1}, \ldots, i_{k}\right\}} \cdot \delta_{0:\left\{i_{1}, \ldots, i_{k}\right\}}<0$ and $C_{\left\{i_{1}, \ldots, i_{k}\right\}} \cdot \delta_{0: J}=0$ for $|J| \leq k$ and $J \neq\left\{i_{1}, \ldots, i_{k}\right\}$. Again from [Rulla 2001, Lemma 1.4.6], the cone generated by the classes $\delta_{0: J}$ for $|J| \leq k$ is thus extremal in $\overline{\operatorname{Eff}}^{1}\left(\overline{\mathcal{M}}_{1, n}\right)$.

Finally, let us consider the class $\delta_{0:\{1, \ldots, n\}}$ for $n \geq 2$. Consider the one-dimensional family $E$ obtained by attaching a rational curve containing all marked points to a base point of a pencil of plane cubics. One has $E \cdot \delta_{0:\{1, \ldots, n\}}<0$ and $E \cdot \delta_{0: J}=0$ for $|J|<n$. Hence, the cone generated by $\delta_{0:\{1, \ldots, n\}}$ and $\delta_{0: J}$ with $|J|<n$ is extremal in $\overline{\operatorname{Eff}}^{1}\left(\overline{\mathcal{M}}_{1, n}\right)$.

We are now ready to prove Theorem 3.

Proof of Theorem 3. Singular elements in $\overline{\mathcal{T}}_{1, n}^{k}$ do not have rational tails. Indeed, consider a singular pointed curve $\left[C, p_{1}, \ldots, p_{n}\right]$ inside the closure of $\mathcal{T o r}_{1, n}^{k}$. The condition $k p_{i} \sim k p_{j}$ means that there exists an admissible cover $\pi: C \rightarrow \mathbb{P}^{1}$ of degree $k$ totally ramified at $p_{i}$ and $p_{j}$. Suppose $C$ has a rational tail $R$ containing $p_{i}$ and $p_{j}$. By the Riemann-Hurwitz formula, $R$ does not contain any other ramification point of $\pi$. Since $\overline{C \backslash R}$ has arithmetic genus 1 , one has $\operatorname{deg}(\pi \mid \overline{C \backslash R})>1$. Hence, the tail $R$ has to meet the other components of $C$ in more than one point, a contradiction. It follows that $\left[\overline{\mathcal{T o r}}_{1, n}^{k}\right]$ has zero intersection with all divisor classes $\delta_{0: J}$. Note that by the projection formula relative to the natural map $\overline{\mathcal{M}}_{1, n} \rightarrow \overline{\mathcal{M}}_{1,1}$, the class $\left[\overline{\mathcal{T} o r}_{1, n}^{k}\right]$ has positive intersection with the divisor class $\lambda$.

From Lemma 4, the cone generated by the classes $\delta_{0: J}$ is extremal in $\overline{\operatorname{Eff}}^{1}\left(\overline{\mathcal{M}}_{1, n}\right)$, hence by duality the class $\left[\overline{\mathcal{T}}_{1, n}^{k}\right]$ spans an extremal ray of $\operatorname{Nef}^{n-1}\left(\overline{\mathcal{M}}_{1, n}\right)$, and this ray does not depend on $k$.

\section{On hyperelliptic loci}

In the following, we collect some well-known facts about classes of hyperelliptic loci which we will use later. For $g \geq 2$ and $0 \leq n \leq 2 g+2$, let

$\mathcal{H} y p_{g, n}:=\left\{\left[C, p_{1}, \ldots, p_{n}\right] \in \mathcal{M}_{g, n} \mid C\right.$ is hyperelliptic

$$
\text { and } \left.h^{0}\left(C, \mathcal{O}_{C}\left(2 p_{i}\right)\right) \geq 2 \text {, for } i=1, \ldots, n\right\}
$$

be the locus of hyperelliptic curves of genus $g$ with $n$ marked Weierstrass points. The locus $\mathcal{H} y p_{g, n}$ has codimension $g-2+n$ in the moduli space $\mathcal{M}_{g, n}$ of smooth curves of genus $g$ with $n$ marked points. The class of the closure $\overline{\mathcal{H y p}}_{g, n}$ is tautological on the moduli of stable curves $\overline{\mathcal{M}}_{g, n}$ [Faber and Pandharipande 2005].

Let $\mathcal{M}_{g, n}^{\mathrm{rt}}$ be the moduli space of curves with rational tails. From [Faber and Pandharipande 2005] or [Graber and Vakil 2005], the tautological group $R^{g-2+n}\left(\mathcal{M}_{g, n}^{\mathrm{rt}}\right)$ is one-dimensional. When $n=0, R^{g-2}\left(\mathcal{M}_{g}\right)$ is one-dimensional and is generated by the class of the hyperelliptic locus $\mathcal{H} y p_{g}$, or equivalently the class $\kappa_{g-2}$ [Looijenga 1995; Faber 1999]. Let $\mathcal{H} y p_{g, n}^{\mathrm{rt}}$ be the restriction of $\overline{\mathcal{H} y p}_{g, n}$ to $\mathcal{M}_{g, n}^{\mathrm{rt}}$. Since the 
pushforward of $\left[\mathcal{H} y p_{g, n}^{\mathrm{rt}}\right]$ via the natural map $\mathcal{M}_{g, n}^{\mathrm{rt}} \rightarrow \mathcal{M}_{g}$ is a positive multiple of $\left[\mathcal{H} y p_{g}\right]$, it follows that $\left[\mathcal{H} y p_{g, n}^{\mathrm{rt}}\right]$ is nonzero and generates $R^{g-2+n}\left(\mathcal{M}_{g, n}^{\mathrm{rt}}\right)$.

Equivalently, $R^{g-2+n}\left(\mathcal{M}_{g, n}^{\mathrm{rt}}\right)$ is generated by the decorated class $\delta_{g, \psi^{g-1}}$ defined in the following way. Consider the gluing map

$$
\xi: \overline{\mathcal{M}}_{g, 1} \rightarrow \overline{\mathcal{M}}_{g, n}
$$

obtained by attaching a chain of $n-1$ rational components at the marked point of an element in $\overline{\mathcal{M}}_{g, 1}$. We fix the markings in an increasing order, from the inner rational component to the outer one. From the rational equivalence of points in $\overline{\mathcal{M}}_{0, n+1}$, the classes of the loci obtained by permuting the markings on the image of $\xi$ are all rationally equivalent. The class $\delta_{g, \psi^{g-1}}$ is defined as the pushforward of the class $\psi^{g-1}$ in $R^{g-1}\left(\overline{\mathcal{M}}_{g, 1}\right)$ via the map $\xi$.

Let $\pi: \overline{\mathcal{M}}_{g, 1} \rightarrow \overline{\mathcal{M}}_{g}$ and $\pi_{n}: \overline{\mathcal{M}}_{g, n} \rightarrow \overline{\mathcal{M}}_{g, n-1}$ be the natural maps. Note that $\left(\pi_{n}\right)_{*} \delta_{g, \psi^{g-1}}=\delta_{g, \psi^{g-1}}$ in $R^{g-2+n-1}\left(\mathcal{M}_{g, n-1}\right)$ for $n \geq 3$, and $\left(\pi_{2}\right)_{*} \delta_{g, \psi^{g-1}}=\psi^{g-1}$. Since $\kappa_{g-2}:=\pi_{*}\left(\psi^{g-1}\right)$ is nonzero in $R^{g-2}\left(\mathcal{M}_{g}\right)$, we conclude $\delta_{g, \psi^{g-1}}$ is nonzero in $R^{g-2+n}\left(\mathcal{M}_{g, n}^{\mathrm{rt}}\right)$.

Example. In the case $g=2$, for $2 \leq n \leq 6$ we have

$$
\left[\mathcal{H} y p_{2, n}^{\mathrm{rt}}\right]=\frac{6 !}{2 \cdot(6-n) !} \delta_{2, \psi} \in R^{n}\left(\mathcal{M}_{2, n}^{\mathrm{rt}}\right) .
$$

Indeed, let us write $\left[\mathcal{H} y p_{2, n}^{\mathrm{rt}}\right]=\alpha \delta_{2, \psi}$ in $R^{n}\left(\mathcal{M}_{2, n}^{\mathrm{rt}}\right)$. In order to determine the coefficient $\alpha$, we intersect both sides of the equation with a test space. Let $C$ be a smooth curve of genus 2, and let $C[n]$ be the $n$-th Fulton-MacPherson compactification of the space of $n$ distinct points of $C$ [Fulton and MacPherson 1994]. The natural map $C[n+1] \rightarrow C[n]$ gives an $n$-dimensional family of genus-two curves with rational tails. Weierstrass points on $C$ are ramification points of the hyperelliptic double covering. Analyzing the Hurwitz space of admissible double coverings, it is easy to see that the intersection $\left[\mathcal{H} y p_{2, n}^{\mathrm{rt}}\right] \cdot C[n]$ corresponds to all ordered $n$-tuples of Weierstrass points in $C$, and is transversal. We deduce that $\left[\mathcal{H} y p_{2, n}^{\mathrm{rt}}\right] \cdot C[n]=6 ! /(6-n)$ !. On the other hand, one has $\delta_{2, \psi} \cdot C[n]=\psi \cdot \xi^{*}(C[n])=\psi \cdot C[1]=2$, whence the statement.

\section{A recursive argument}

Let $N^{k}\left(\overline{\mathcal{M}}_{g, n}\right)$ be the group of codimension- $k$ cycles on $\overline{\mathcal{M}}_{g, n}$ modulo numerical equivalence. We denote by $\operatorname{Eff}^{k}\left(\overline{\mathcal{M}}_{g, n}\right) \subset N^{k}\left(\overline{\mathcal{M}}_{g, n}\right)$ the cone of effective cycle classes, and by $\operatorname{REff}^{k}\left(\overline{\mathcal{M}}_{g, n}\right) \subseteq \operatorname{Eff}^{k}\left(\overline{\mathcal{M}}_{g, n}\right)$ the subcone of effective tautological classes (see [Faber and Pandharipande 2005] for tautological classes on $\overline{\mathcal{M}}_{g, n}$ ).

A cycle class $E$ inside a cone $K \subset N^{k}\left(\overline{\mathcal{M}}_{g, n}\right)$ is called extremal in $K$ if whenever two cycle classes $E_{1}$ and $E_{2}$ in $K$ are such that $E=E_{1}+E_{2}$, then both $E_{1}$ and 
$E_{2}$ lie in the ray spanned by $E$. An effective cycle class $E$ is called rigid if any effective cycle with class $m E$ is supported on the support of $E$.

Theorem 5. Given $g \geq 2$, if $\left[\overline{\mathcal{H} y p}_{g, 2}\right]$ is rigid and extremal in $\operatorname{REff}^{g}\left(\overline{\mathcal{M}}_{g, 2}\right)$, then $\left[\overline{\mathcal{H} y p}_{g, n}\right]$ is rigid and extremal in REff $^{g-2+n}\left(\overline{\mathcal{M}}_{g, n}\right)$, for $3 \leq n \leq 2 g+2$.

Proof. Let $n \geq 3$ and assume that the statement is true for $\overline{\mathcal{H} y p}_{g, n-1}$. Suppose that

$$
\left[\overline{\mathcal{H} y p}_{g, n}\right]=\sum_{i} a_{i}\left[X_{i}\right]
$$

with $a_{i}>0, X_{i}$ irreducible, tautological, effective of codimension $n$, and $\left[X_{i}\right]$ not proportional to $\left[\overline{\mathcal{H} y p}_{g, n}\right]$, for all $i$.

Since $R^{g-2+n}\left(\mathcal{M}_{g, n}^{\mathrm{rt}}\right)$ is generated by $\delta_{g, \psi^{g-1}}$ (see Section 2), we can express the class of each $X_{i}$ as

$$
\left[X_{i}\right]=c_{i} \delta_{g, \psi^{g-1}}+B_{i},
$$

where $c_{i}$ is a nonnegative coefficient, and $B_{i}$ is a (not necessarily effective) cycle class in $R^{g-2+n}\left(\overline{\mathcal{M}}_{g, n}\right)$ with $B_{i}=0$ in $R^{g-2+n}\left(\mathcal{M}_{g, n}^{\text {rt }}\right)$. Let $\pi_{j}: \overline{\mathcal{M}}_{g, n} \rightarrow \overline{\mathcal{M}}_{g, n-1}$ be the map obtained by forgetting the point $j$. Applying $\left(\pi_{j}\right)_{*}$ to $(1)$, we have

$$
(2 g+2-(n-1))\left[\overline{\mathcal{H} y p}_{g, n-1}\right]=\sum_{i} a_{i}\left(\pi_{j}\right)_{*}\left[X_{i}\right] .
$$

Pick a locus $X_{i}$ appearing on the right side of (1). Consider two cases. First, suppose $\left(\pi_{1}\right)_{*}\left[X_{i}\right]=\cdots=\left(\pi_{n}\right)_{*}\left[X_{i}\right]=0$. Note that $\left(\pi_{j}\right)_{*} \delta_{g, \psi^{g-1}}=\delta_{g, \psi^{g-1}}$ in $R^{g-2+n-1}\left(\mathcal{M}_{g, n-1}^{\mathrm{rt}}\right)$, for all $j=1, \ldots, n$. Since $B_{i}=0$ in $R^{g-2+n}\left(\mathcal{M}_{g, n}^{\mathrm{rt}}\right)$, using the exact sequence

$$
A^{g-2+n}\left(\overline{\mathcal{M}}_{g, n} \backslash \mathcal{M}_{g, n}^{\mathrm{rt}}\right) \rightarrow A^{g-2+n}\left(\overline{\mathcal{M}}_{g, n}\right) \rightarrow A^{g-2+n}\left(\mathcal{M}_{g, n}^{\mathrm{rt}}\right) \rightarrow 0,
$$

we can assume that $B_{i}$ is represented by a linear combination of cycle classes supported in $\overline{\mathcal{M}}_{g, n} \backslash \mathcal{M}_{g, n}^{\mathrm{rt}}$. An element in the support of such a cycle does not have an irreducible and smooth component of genus $g$, hence $\left(\pi_{j}\right)_{*} B_{i}=0$ in $A^{g-2+n-1}\left(\mathcal{M}_{g, n-1}^{\mathrm{rt}}\right)$, for all $j=1, \ldots, n$. We deduce that $c_{i}=0$, that is, $\left[X_{i}\right]=0$ in $R^{g-2+n}\left(\mathcal{M}_{g, n}^{\mathrm{rt}}\right)$.

For the other case, suppose $\left(\pi_{1}\right)_{*}\left[X_{i}\right]$ is nonzero. Since the class $\left[\overline{\mathcal{H} y p}_{g, n-1}\right]$ is rigid and extremal in REff ${ }^{g+n-3}\left(\overline{\mathcal{M}}_{g, n-1}\right)$, from (2) we deduce that $\left(\pi_{1}\right)_{*}\left[X_{i}\right]$ is a positive multiple of the class of $\overline{\mathcal{H} y p}_{g, n-1}$ and, moreover, $X_{i} \subset\left(\pi_{1}\right)^{-1} \overline{\mathcal{H} y p}_{g, n-1}$. This implies that $\left(\pi_{2}\right)_{*}\left[X_{i}\right], \ldots,\left(\pi_{n}\right)_{*}\left[X_{i}\right]$ are also nonzero. It follows that $X_{i}$ is in the intersection of all the $\left(\pi_{j}\right)^{-1} \overline{\mathcal{H} y p}_{g, n-1}$, for $j=1, \ldots, n$. In particular, any $n-1$ marked points in a general element of $X_{i}$ are distinct Weierstrass points. Hence, all $n$ marked points must be distinct Weierstrass points. (Note that $n \geq 3$.) This forces $\left[X_{i}\right]$ to be a positive multiple of $\left[\overline{\mathcal{H} y p}_{g, n}\right]$, a contradiction. 
Finally, the above steps show that $\left[X_{i}\right]=0$ in $R^{g-2+n}\left(\mathcal{M}_{g, n}^{\mathrm{rt}}\right)$, for all $i$. This yields a contradiction since $\left[\overline{\mathcal{H} y p}_{g, n}\right] \neq 0$ in $R^{g-2+n}\left(\mathcal{M}_{g, n}^{\mathrm{rt}}\right)$ (see Section 2), hence $\left[\overline{\mathcal{H} y p}_{g, n}\right]$ is extremal in $\operatorname{REff}^{g-2+n}\left(\overline{\mathcal{M}}_{g, n}\right)$.

Suppose that $E:=m\left[\overline{\mathcal{H} y p}_{g, n}\right]$ is effective. Since

$$
\left(\pi_{j}\right)_{*}(E)=(2 g-n+3) m\left[{\overline{\mathcal{H}} y p_{g, n-1}}\right],
$$

and since $\left[\overline{\mathcal{H} y p}_{g, n-1}\right]$ is rigid, $\left(\pi_{j}\right)_{*}(E)$ is supported on $\overline{\mathcal{H} y p}_{g, n-1}$, for $j=1, \ldots, n$. This implies that $E$ is supported on the intersection of all the $\left(\pi_{j}\right)^{-1} \overline{\mathcal{H} y p}_{g, n-1}$, for $j=1, \ldots, n$. We conclude that $E$ is supported on $\overline{\mathcal{H} y p}_{g, n}$ and $\left[\overline{\mathcal{H} y p}_{g, n}\right]$ is rigid.

Remark 6. The classes $\left[\overline{\mathcal{H} y p}_{3}\right],\left[\overline{\mathcal{H} y p}_{3,1}\right]$, and $\left[\overline{\mathcal{H} y p}_{4}\right]$ are known to be extremal in $\operatorname{Eff}^{1}\left(\overline{\mathcal{M}}_{3}\right)$ [Rulla 2001], $\operatorname{Eff}^{2}\left(\overline{\mathcal{M}}_{3,1}\right)$, and $\operatorname{Eff}^{2}\left(\overline{\mathcal{M}}_{4}\right)$ [Chen and Coskun 2015], respectively. It is natural to wonder whether $\left[\overline{\mathcal{H} y p}_{g, n}\right]$ is extremal in $\operatorname{REff}^{g-2+n}\left(\overline{\mathcal{M}}_{g, n}\right)$, for all $g \geq 2$ and $0 \leq n \leq 2 g+2$. By Theorem 5, it is enough to study the cases $n \leq 2$.

\section{Loci of Weierstrass points on curves of genus 2}

In this section, we complete the proof of Theorem 1. It is enough to show that $\left[\overline{\mathcal{H} y p}_{2, n}\right]$ is rigid and extremal in $\operatorname{REff}^{n}\left(\overline{\mathcal{M}}_{2, n}\right)$, and to use the fact that for small values of $n$, REff* $\left(\overline{\mathcal{M}}_{2, n}\right)=\operatorname{Eff}^{*}\left(\overline{\mathcal{M}}_{2, n}\right)$. Indeed, according to [Petersen and Tommasi 2014; Petersen 2016, Theorem 3.8], all even cohomology of $\overline{\mathcal{M}}_{2, n}$ is tautological for $n<20$. Note that the Betti numbers of $\overline{\mathcal{M}}_{2, n}$ for $n \leq 7$ have been computed in [Getzler 1998; Bergström 2009].

4.1. The classes for $n=1,2$. When $n=1$, the class of the divisor $\overline{\mathcal{H} y p}_{2,1}$ in $\overline{\mathcal{M}}_{2,1}$ is

$$
\left[\overline{\mathcal{H} y p}_{2,1}\right]=3 \omega-\frac{1}{10} \delta_{\text {irr }}-\frac{6}{5} \delta_{1}=3 \omega-\lambda-\delta_{1} \in \operatorname{Pic}\left(\overline{\mathcal{M}}_{2,1}\right)
$$

[Eisenbud and Harris 1987, Theorem 2.2], and [ $\left.\overline{\mathcal{H} y p}_{2,1}\right]$ is rigid and extremal in $\operatorname{Eff}^{1}\left(\overline{\mathcal{M}}_{2,1}\right)$ [Rulla 2001]. When $n=2$, the class of the double ramification locus $\overline{\mathcal{H} y p}_{2,2}$ in $\overline{\mathcal{M}}_{2,2}$ is

$$
\begin{aligned}
{\left[\overline{\mathcal{H} y p}_{2,2}\right]=6 \psi_{1} \cdot \psi_{2}-\frac{3}{2}(} & \left.\psi_{1}^{2}+\psi_{2}^{2}\right) \\
& -\left(\psi_{1}+\psi_{2}\right) \cdot\left(\frac{21}{10} \delta_{1: 1}+\frac{3}{5} \delta_{1: 0}+\frac{1}{20} \delta_{\text {irr }}\right) \in A^{2}\left(\overline{\mathcal{M}}_{2,2}\right)
\end{aligned}
$$

and $\overline{\mathcal{H} y p}_{2,2}$ is not a complete intersection [Tarasca 2015]. Expressing products of divisor classes in terms of decorated boundary strata classes, we have

$$
\left[\overline{\mathcal{H} y p}_{2,2}\right]=5 \delta_{2, w}+9 \delta_{11 \mid}+\frac{5}{8} \delta_{01 \mid}-\frac{1}{8}\left(\delta_{01 \mid 1}+\delta_{01 \mid 2}+\delta_{01 \mid 12}\right)+2 \gamma_{1: \varnothing}+\frac{1}{24} \delta_{00} .
$$

Here, $\delta_{2, w}$ is the class of the locus of curves with a rational tail containing both marked points attached at a Weierstrass point on a component of genus $2 ; \delta_{11 \mid}$ is the class of the locus of curves whose general element has two elliptic tails attached at a rational component containing both marked points; $\delta_{01 \mid}, \delta_{01 \mid 1}, \delta_{01 \mid 2}, \delta_{01 \mid 12}$ are the 
classes of the loci of curves whose general element has an elliptic tail attached at a nodal rational component with the points distributed in the following way: for the class $\delta_{01 \mid}$ both marked points are on the rational component, for $\delta_{01 \mid i}$ the point $i$ is on the elliptic component and the other marked point is on the rational component, and for $\delta_{01 \mid 12}$ both marked points are on the elliptic component; $\gamma_{1: \varnothing}$ is the class of the locus of curves with an elliptic component meeting in two points a rational component containing both marked points; and finally, $\delta_{00}$ is the class of the locus whose general element is a rational curve with two nondisconnecting nodes.

In Section 4.2, we will use in a crucial way the expression in (5). In Section 5, we will also use the following description. Let $\pi_{i}: \overline{\mathcal{M}}_{2,2} \rightarrow \overline{\mathcal{M}}_{2,1}$ be the map obtained by forgetting the point $i$, for $i=1,2$.

Lemma 7. The following equality holds in $A^{2}\left(\overline{\mathcal{M}}_{2,2}\right)$ :

$$
\pi_{1}^{*}\left[\overline{\mathcal{H} y p}_{2,1}\right] \cdot \pi_{2}^{*}\left[\overline{\mathcal{H} y p}_{2,1}\right]=\left[\overline{\mathcal{H} y p}_{2,2}\right]+\gamma_{1: \varnothing}+\delta_{2, w} .
$$

In particular, $\pi_{1}^{-1}\left(\overline{\mathcal{H} y p}_{2,1}\right) \cap \pi_{2}^{-1}\left(\overline{\mathcal{H} y p}_{2,1}\right)$ is the union of the supports of $\left[\overline{\mathcal{H} y p}_{2,2}\right]$, $\gamma_{1: \varnothing}$, and $\delta_{2, w}$.

Proof. The desired equality follows from (3) and (4). Since the supports of [ $\left.\overline{\mathcal{H} y p}_{2,2}\right]$, $\gamma_{1: \varnothing}$, and $\delta_{2, w}$ are contained in $\pi_{i}^{-1}\left(\overline{\mathcal{H} y p}_{2,1}\right)$, for $i=1,2$, the statement follows.

Note that

$$
\delta_{2, w}=\delta_{0: 2} \cdot \pi_{1}^{*}\left[\overline{\mathcal{H} y p}_{2,1}\right]=\delta_{0: 2} \cdot\left(3 \omega_{1}-\lambda-\delta_{1}\right) .
$$

Hence, we can write

$$
\begin{aligned}
{\left[\overline{\mathcal{H} y p}_{2,2}\right] } & =\pi_{1}^{*}\left(3 \omega_{2}-\lambda-\delta_{1}\right) \cdot \pi_{2}^{*}\left(3 \omega_{1}-\lambda-\delta_{1}\right)-\delta_{0: 2} \cdot\left(3 \omega_{1}-\lambda-\delta_{1}\right)-\gamma_{1: \varnothing} \\
& =\left(3 \omega_{2}-\lambda-\delta_{1}\right) \cdot\left(3 \omega_{1}-\lambda-\delta_{1}\right)-\delta_{0: 2} \cdot\left(3 \omega_{1}-\lambda-\delta_{1}\right)-\gamma_{1: \varnothing} .
\end{aligned}
$$

4.2. The extremality for $\boldsymbol{n}=2$. By Theorem 5 , in order to show that $\left[\overline{\mathcal{H} y p}_{2, n}\right]$ is rigid and extremal in $\operatorname{REff}^{n}\left(\overline{\mathcal{M}}_{2, n}\right)$ for $2 \leq n \leq 6$, it is enough to show that $\left[\overline{\mathcal{H}} y p_{2,2}\right]$ is rigid and extremal in $\operatorname{Eff}^{2}\left(\overline{\mathcal{M}}_{2,2}\right)$.

Theorem 8. $\left[\overline{\mathcal{H} y p}_{2,2}\right]$ is rigid and extremal in $\operatorname{Eff}^{2}\left(\overline{\mathcal{M}}_{2,2}\right)$.

Proof. Suppose that

$$
\left[\overline{\mathcal{H} y p}_{2,2}\right]=\sum_{i} a_{i}\left[X_{i}\right]
$$

where $a_{i}>0$ and $X_{i}$ is an irreducible codimension-two effective cycle on $\overline{\mathcal{M}}_{2,2}$ with $\left[X_{i}\right]$ not proportional to $\left[\overline{\mathcal{H} y p}_{2,2}\right]$, for all $i$. Let $\pi_{j}: \overline{\mathcal{M}}_{2,2} \rightarrow \overline{\mathcal{M}}_{2,1}$ be the map forgetting the point $j$, for $j=1,2$, and $\pi: \overline{\mathcal{M}}_{2,2} \rightarrow \overline{\mathcal{M}}_{2}$ be the map forgetting both marked points. Applying $\left(\pi_{j}\right)_{*}$ to $(7)$, we obtain

$$
5\left[\overline{\mathcal{H} y p}_{2,1}\right]=\sum_{i} a_{i}\left(\pi_{1}\right)_{*}\left[X_{i}\right] .
$$


Pick a locus $X_{i}$ appearing on the right side of (7). If $\left(\pi_{1}\right)_{*}\left[X_{i}\right]=\left(\pi_{2}\right)_{*}\left[X_{i}\right]=0$, then either $X_{i}$ is contained in the inverse image via $\pi$ of a codimension-two effective cycle on $\overline{\mathcal{M}}_{2}$, or a general point of $X_{i}$ contains a smooth rational component with two marked points and two singular points. Note that a codimension-two locus in $\overline{\mathcal{M}}_{2}$ is a curve, and the cone of effective curves in $\overline{\mathcal{M}}_{2}$ is known to be spanned by the two one-dimensional boundary strata. We deduce that $\left[X_{i}\right]$ is in the cone generated by the boundary strata classes $\delta_{01 \mid}, \delta_{01 \mid 1}, \delta_{01 \mid 2}, \delta_{01 \mid 12}, \delta_{00}, \delta_{11 \mid}$, and $\gamma_{1: \varnothing}$.

Suppose $\left(\pi_{1}\right)_{*}\left[X_{i}\right]$ is nonzero. Since $\left[\overline{\mathcal{H} y p}_{2,1}\right]$ is rigid and extremal in $\operatorname{Eff}^{1}\left(\overline{\mathcal{M}}_{2,1}\right)$, from (8) we deduce that $X_{i} \subset \pi_{1}^{-1}\left(\overline{\mathcal{H} y p}_{2,1}\right)$. Hence, $\left(\pi_{2}\right)_{*}\left[X_{i}\right]$ is also nonzero, and $X_{i} \subset \pi_{1}^{-1}\left(\overline{\mathcal{H} y p}_{2,1}\right) \cap \pi_{2}^{-1}\left(\overline{\mathcal{H} y p}_{2,1}\right)$. From Lemma 7 we conclude that $\left[X_{i}\right]$ is supported on the locus of curves with a rational tail containing both marked points attached at a Weierstrass point of a genus-two curve, hence $\left[X_{i}\right]$ is a positive multiple of $\delta_{2, w}$.

From (5), the class of $\overline{\mathcal{H} y p}_{2,2}$ lies outside the cone generated by $\delta_{2, w}, \delta_{01 \mid}, \delta_{01 \mid 1}$, $\delta_{01 \mid 2}, \delta_{01 \mid 12}, \delta_{00}, \delta_{11 \mid}$, and $\gamma_{1: \varnothing}$. Indeed, the coefficient of $\delta_{01 \mid 1}+\delta_{01 \mid 2}+\delta_{01 \mid 12}$ is negative. Hence $\left[\overline{\mathcal{H} y p}_{2,2}\right]$ is extremal in $\operatorname{Eff}^{2}\left(\overline{\mathcal{M}}_{2,2}\right)$.

The rigidity of $\left[\overline{\mathcal{H} y p}_{2,2}\right]$ follows from a similar argument. Suppose that for some positive $m, E:=m\left[\overline{\mathcal{H} y p}_{2,2}\right]$ is effective. Since $\left(\pi_{j}\right)_{*}(E)=5 m\left[\overline{\mathcal{H} y p}_{2,1}\right]$ and $\left[\overline{\mathcal{H} y p}_{2,1}\right]$ is rigid, we have that $\left(\pi_{j}\right)_{*}(E)$ is supported on $\overline{\mathcal{H} y p}_{2,1}$, for $j=1,2$. Hence, the support of $E$ is in $\pi_{1}^{-1}\left(\overline{\mathcal{H} y p}_{2,1}\right) \cap \pi_{2}^{-1}\left(\overline{\mathcal{H} y p}_{2,1}\right)$. From Lemma 7, $E$ is supported on the union of the loci ${\overline{\mathcal{H}} y p_{2,2}}_{2}, \Gamma_{1: \varnothing}$, and $\Delta_{2, w}$. Since $E=m\left[\overline{\mathcal{H} y p}_{2,2}\right]$, $E$ is supported only on $\overline{\mathcal{H} y p}_{2,2}$, and the statement follows.

\section{The class of $\overline{\mathcal{H} y p}_{2,3}$}

The aim of this section is to compute the class of $\overline{\mathcal{H} y p}_{2,3}$ in $A^{3}\left(\overline{\mathcal{M}}_{2,3}\right)$. We first discuss a recursive relation between the classes of a partial closure of $\mathcal{H} y p_{2, n}$ and $\mathcal{H} y p_{2, n-1}$.

Recall the map $\pi_{i}: \overline{\mathcal{M}}_{g, n} \rightarrow \overline{\mathcal{M}}_{g, n-1}$ obtained by forgetting the $i$-th marked point, and the map $\rho_{i}: \overline{\mathcal{M}}_{g, n} \rightarrow \overline{\mathcal{M}}_{g, 1}$ obtained by forgetting all but the $i$-th marked point.

5.1. A recursive relation. Let $\overline{\mathcal{M}}_{g, n}^{o}$ be the open locus in $\overline{\mathcal{M}}_{g, n}$ of stable curves with at most one nondisconnecting node. Let $\overline{\mathcal{H} y p}_{g, n}^{o}$ be the closure of $\mathcal{H} y p_{g, n}$ in $\overline{\mathcal{M}}_{g, n}^{o}$. For $2 \leq n \leq 6$, we note the following identity in $A^{n}\left(\overline{\mathcal{M}}_{2, n}^{o}\right)$ :

$$
\pi_{n}^{*}\left(\overline{\mathcal{H} y p}_{2, n-1}^{o}\right) \cdot \rho_{n}^{*}\left(\overline{\mathcal{H} y p}_{2,1}\right) \equiv \overline{\mathcal{H} y p}_{2, n}^{o}+\sum_{i=1}^{n-1} \pi_{n}^{*}\left(\overline{\mathcal{H} y p}_{2, n-1}^{o}\right) \cdot \delta_{0:\{i, n\}} .
$$

Indeed, the intersection on the left-hand side consists of genus-two curves with a choice of $n$ ordered Weierstrass points, the first $n-1$ being distinct. The component $\overline{\mathcal{H} y p}_{2, n}^{o}$ corresponds to curves with all $n$ points distinct, and the component $\pi_{n}^{*}\left(\overline{\mathcal{H} y p}_{2, n-1}^{o}\right) \cdot \delta_{0:\{i, n\}}$ corresponds to curves with the $n$-th point coinciding with 
the $i$-th point, for $i=1, \ldots, n-1$. A Weierstrass point on a smooth hyperelliptic curve of genus $g$ has weight $g(g-1) / 2$. This explains the coefficient of $\overline{\mathcal{H y p}}_{2, n}^{o}$. Since the right-hand side is symmetric with respect to the first $n-1$ points, it is clear that all the components $\pi_{n}^{*}\left(\overline{\mathcal{H} y p}_{2, n-1}^{o}\right) \cdot \delta_{0:\{i, n\}}$ have equal multiplicity, which, forgetting the point $n$, must equal 1 .

Using (9), one can recursively express the class of $\overline{\mathcal{H} y p}_{2, n}^{o}$ in terms of products of divisor classes. In the following, we derive a complete expression for the class of $\overline{\mathcal{H} y p}_{2,3}$ in $A^{3}\left(\overline{\mathcal{M}}_{2,3}\right)$.

5.2. A set-theoretic description. To extend (9) with $n=3$ over $\overline{\mathcal{M}}_{2,3}$, we need to consider loci of curves with at least two nondisconnecting nodes. Let $\Xi_{i}$ be the closure of the locus of curves with an elliptic component $\left[E, p_{i}, x, y\right]$ such that $2 p_{i} \sim x+y$, and a rational component containing the other two marked points $p_{j}, p_{k}$, and meeting $E$ at the points $x, y$. Let $\Theta$ be the closure of the locus of curves whose general element has a rational component $\left[R, p_{1}, p_{2}, p_{3}, x, y\right]$ such that $2 p_{1} \sim 2 p_{2} \sim x+y$, and an elliptic component meeting $R$ at the points $x, y$.

\section{Proposition 9. We have}

$$
\begin{aligned}
\pi_{3}^{*}\left(\overline{\mathcal{H} y p}_{2,2}\right) & \cap \rho_{3}^{*}\left(\overline{\mathcal{H} y p}_{2,1}\right) \\
& =\overline{\mathcal{H} y p}_{2,3} \cup \pi_{3}^{*}\left(\overline{\mathcal{H} y p}_{2,2}\right)_{\mid \Delta_{0:\{1,3\}}} \cup \pi_{3}^{*}\left(\overline{\mathcal{H} y p}_{2,2}\right)_{\mid \Delta_{0:\{2,3\}}} \cup \Xi_{1} \cup \Xi_{2} \cup \Theta .
\end{aligned}
$$

Proof. The intersection

$$
\pi_{3}^{*}\left(\overline{\mathcal{H} y p}_{2,2}\right) \cap \rho_{3}^{*}\left(\overline{\mathcal{H} y p}_{2,1}\right)
$$

consists of stable curves $\left[C, p_{1}, p_{2}, p_{3}\right]$ with three marked Weierstrass points and with $p_{1}$ and $p_{2}$ corresponding to two different Weierstrass points. If the three points correspond to three different Weierstrass points, then $\left[C, p_{1}, p_{2}, p_{3}\right]$ is in $\overline{\mathcal{H} y p}_{2,3}$. If $p_{3}$ and $p_{1}$ correspond to the same Weierstrass point, then $\left[C, p_{1}, p_{2}, p_{3}\right]$ is in the restriction of $\pi_{3}^{*}\left(\overline{\mathcal{H} y p}_{2,2}\right)$ to $\Delta_{0:\{1,3\}}$. The case when $p_{3}$ and $p_{2}$ correspond to the same Weierstrass point is similar. Finally, restricting the intersection to the codimension-two boundary strata and using admissible covers to describe Weierstrass points on singular curves, we deduce that $\Xi_{1}, \Xi_{2}$, and $\Theta$ are the only additional components contained in the intersection, hence the statement.

5.3. The multiplicities. Since the left-hand side of the expression in Proposition 9 is symmetric with respect to the first two marked points, we conclude that the contributions of $\pi_{3}^{*}\left[\overline{\mathcal{H} y p}_{2,2}\right] \cdot \delta_{0:\{1,3\}}$ and $\pi_{3}^{*}\left[\overline{\mathcal{H} y p}_{2,2}\right] \cdot \delta_{0:\{2,3\}}$ on the right-hand side are equal. Similarly for $\Xi_{1}$ and $\Xi_{2}$. Hence we have, for some coefficients $\alpha, \beta, \gamma, \delta$,

$$
\begin{aligned}
& \pi_{3}^{*}\left[\overline{\mathcal{H} y p}_{2,2}\right] \cdot \rho_{3}^{*}\left[\overline{\mathcal{H} y p}_{2,1}\right] \\
& =\alpha\left[\overline{\mathcal{H} y p}_{2,3}\right]+\beta\left(\delta_{0:\{1,3\}}+\delta_{0:\{2,3\}}\right) \cdot \pi_{3}^{*}\left[\overline{\mathcal{H} y p}_{2,2}\right]+\gamma\left(\left[\Xi_{1}\right]+\left[\Xi_{2}\right]\right)+\delta[\Theta] .
\end{aligned}
$$




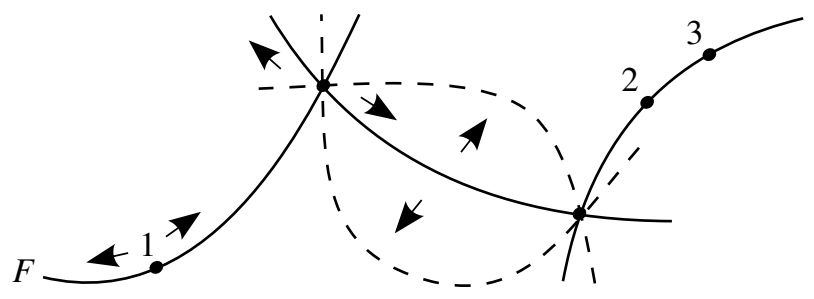

Figure 1. How the general element of the family moves.

Forgetting the first marked point in (10), the left-hand side is

$$
5\left(\pi_{1}^{*}\left[\overline{\mathcal{H} y p}_{2,1}\right] \cdot \pi_{2}^{*}\left[\overline{\mathcal{H} y p}_{2,1}\right]\right)=5\left(\left[\overline{\mathcal{H} y p}_{2,2}\right]+\gamma_{1: \varnothing}+\delta_{2, w}\right)
$$

by Lemma 7, hence we have

$$
5\left(\left[\overline{\mathcal{H} y p}_{2,2}\right]+\gamma_{1: \varnothing}+\delta_{2, w}\right)=(4 \alpha+\beta)\left[\overline{\mathcal{H} y p}_{2,2}\right]+(4 \gamma+\delta) \gamma_{1: \varnothing}+5 \beta \delta_{2, w} .
$$

We deduce $\alpha=\beta=1$ and $4 \gamma+\delta=5$.

In order to compute $\gamma$ and $\delta$, we consider the restriction of (10) to the following three-dimensional test family. Attach at two points of an elliptic curve $E$ a rational tail containing the points marked by 2 and 3, and an elliptic tail $F$ containing the point marked by 1 . Consider the family obtained by varying $E$ in a pencil of degree 12 , by varying the point of intersection with the rational tail on the central elliptic component in which it lies, and by varying the point marked by 1 on $F$ (see Figure 1). The base of this family is $Y \times F$, where $Y$ is the blow-up of $\mathbb{P}^{2}$ at the nine points of intersection of two general cubics.

Let $H$ be the pullback of the hyperplane class in $\mathbb{P}^{2}$, let $\Sigma$ be the sum of the nine exceptional divisors, and let $E_{0}$ be one of them. Denote by $\pi: Y \times F \rightarrow F$ the natural projection, and let $q=E \cap F$ be the singular point on $F$. The divisor classes on $\overline{\mathcal{M}}_{2,3}$ restrict as follows:

$$
\begin{aligned}
\psi_{1} & =\pi^{*}(q), \\
\delta_{\text {irr }} & =36 H-12 \Sigma=12 \lambda, \\
\delta_{0:\{2,3\}} & =-3 H+\Sigma-E_{0}, \\
\delta_{1:\{1\}} & =-3 H+\Sigma-E_{0}-\pi^{*}(q), \\
\delta_{1: 0} & =E_{0}+\pi^{*}(q) .
\end{aligned}
$$

From (3) and (4), it follows that

$$
\begin{aligned}
& \rho_{3}^{*}\left[\overline{\mathcal{H} y p}_{2,1}\right] \cdot \pi_{3}^{*}\left[\overline{\mathcal{H} y p}_{2,2}\right]=-\left(3 \delta_{0:\{2,3\}}+\frac{1}{10} \delta_{\text {irr }}+\frac{6}{5}\left(\delta_{1:\{1\}}+\delta_{1: 0}\right)\right) \\
& \quad \cdot\left(-6 \psi_{1} \cdot \delta_{0:\{2,3\}}-\frac{3}{2} \delta_{0:\{2,3\}}^{2}-\left(\psi_{1}-\delta_{0:\{2,3\}}\right) \cdot\left(\frac{21}{10} \delta_{1:\{1\}}+\frac{3}{5} \delta_{1: 0}+\frac{1}{20} \delta_{\text {irr }}\right)\right)=27,
\end{aligned}
$$


and similarly,

$$
\pi_{3}^{*}\left[\overline{\mathcal{H} y p}_{2,2}\right] \cdot \delta_{0:\{2,3\}}=-9 .
$$

Note that this family meets $\Xi_{1}$ when $E$ degenerates to one of the 12 rational nodal fibers, the rational tail is attached at a point colliding with the nondisconnecting node, and the point marked by 1 differs from $q$ in $F$ by a nontrivial torsion point of order 2 in $\mathrm{Pic}^{0}(F)$. The intersection is transverse at each point, hence we have

$$
\Xi_{1}=12 \cdot 3
$$

All other classes in (10) are disjoint from this family. We deduce the relation

$$
27=-9 \beta+36 \gamma
$$

and hence conclude that $\alpha=\beta=\gamma=\delta=1$. We have thus proved the following statement.

Proposition 10. One has

$$
\left[\overline{\mathcal{H} y p}_{2,3}\right]=\pi_{3}^{*}\left[\overline{\mathcal{H} y p}_{2,2}\right] \cdot\left(\rho_{3}^{*}\left[\overline{\mathcal{H} y p}_{2,1}\right]-\delta_{0:\{1,3\}}-\delta_{0:\{2,3\}}\right)-\left[\Xi_{1}\right]-\left[\Xi_{2}\right]-[\Theta] .
$$

5.4. The boundary components. It remains to compute the classes of $\Xi_{1}, \Xi_{2}$, and $\Theta$. Recall the classes $\gamma_{1: J}$ defined in the introduction.

Lemma 11. The following equalities hold in $A^{3}\left(\overline{\mathcal{M}}_{2,3}\right)$ :

$$
\begin{aligned}
& {\left[\Xi_{i}\right]=\left(2 \psi_{i}-\delta_{1:\{i\}}\right) \cdot \gamma_{1:\{i\}} \quad \text { for } i=1,2,} \\
& {[\Theta]=\gamma_{1: \varnothing} \cdot\left(\psi_{1}-\delta_{0:\{1,3\}}\right)=\gamma_{1: \varnothing} \cdot\left(\psi_{2}-\delta_{0:\{2,3\}}\right) .}
\end{aligned}
$$

Proof. Consider the divisor $D_{i}$ of curves $\left[E, p_{i}, x, y\right]$ in $\mathcal{M}_{1,3}$ such that $2 p_{i} \sim x+y$. From [Belorousski and Pandharipande 2000, §2.6] or [Chen and Coskun 2014, Proposition 3.1], one has $\left[\bar{D}_{i}\right]=2 \psi_{i}-\delta_{1:\{i\}}$ in $\operatorname{Pic}\left(\overline{\mathcal{M}}_{1,3}\right)$. The locus $\Xi_{i}$ is the pushforward of $\bar{D}_{i} \times \overline{\mathcal{M}}_{0,4} \subset \overline{\mathcal{M}}_{1,3} \times \overline{\mathcal{M}}_{0,4}$ via the natural map

$$
\overline{\mathcal{M}}_{1,3} \times \overline{\mathcal{M}}_{0,4} \rightarrow \Gamma_{1:\{i\}} \subset \overline{\mathcal{M}}_{2,3} .
$$

Similarly, consider the map $\xi: \overline{\mathcal{M}}_{1,2} \times \overline{\mathcal{M}}_{0,5} \rightarrow \Gamma_{1: \varnothing} \subset \overline{\mathcal{M}}_{2,3}$ defined as

$$
\left(\left[E, x_{1}, y_{1}\right],\left[R, p_{1}, p_{2}, p_{3}, x_{2}, y_{2}\right]\right) \mapsto\left[E \cup_{x_{1} \sim x_{2}, y_{1} \sim y_{2}} R, p_{1}, p_{2}, p_{3}\right] .
$$

The locus $\Theta$ is the pushforward via $\xi$ of the locus $\overline{\mathcal{M}}_{1,2} \times \pi_{3}^{*}$ (point) $\subset \overline{\mathcal{M}}_{1,2} \times \overline{\mathcal{M}}_{0,5}$, where $\pi_{3}: \overline{\mathcal{M}}_{0,5} \rightarrow \overline{\mathcal{M}}_{0,4}$ is the map obtained by forgetting the point $p_{3}$. The statement follows.

From (3), (6), and Lemma 11, the resulting expression in Proposition 10 gives the statement in Theorem 2 . 


\section{Acknowledgements}

We would like to thank Izzet Coskun for helpful discussions on related ideas, Dan Petersen for pointing our attention to the results in [Petersen and Tommasi 2014; Petersen 2016], and the referee for suggesting many improvements on the exposition.

\section{References}

[Belorousski and Pandharipande 2000] P. Belorousski and R. Pandharipande, "A descendent relation in genus 2", Ann. Scuola Norm. Sup. Pisa Cl. Sci. (4) 29:1 (2000), 171-191. MR Zbl

[Bergström 2009] J. Bergström, "Equivariant counts of points of the moduli spaces of pointed hyperelliptic curves", Doc. Math. 14 (2009), 259-296. MR Zbl

[Chen and Coskun 2014] D. Chen and I. Coskun, "Extremal effective divisors on $\bar{M}_{1, n}$ ", Math. Ann. 359:3-4 (2014), 891-908. MR Zbl

[Chen and Coskun 2015] D. Chen and I. Coskun, "Extremal higher codimension cycles on moduli spaces of curves", Proc. Lond. Math. Soc. (3) 111:1 (2015), 181-204. MR Zbl

[Eisenbud and Harris 1987] D. Eisenbud and J. Harris, "The Kodaira dimension of the moduli space of curves of genus $\geq 23$ ", Invent. Math. 90:2 (1987), 359-387. MR Zbl

[Faber 1999] C. Faber, "A conjectural description of the tautological ring of the moduli space of curves", pp. 109-129 in Moduli of curves and abelian varieties, edited by C. Faber and E. Looijenga, Aspects Math. E33, Vieweg, Braunschweig, 1999. MR Zbl

[Faber and Pandharipande 2005] C. Faber and R. Pandharipande, "Relative maps and tautological classes”, J. Eur. Math. Soc. (JEMS) 7:1 (2005), 13-49. MR Zbl

[Fulton and MacPherson 1994] W. Fulton and R. MacPherson, "A compactification of configuration spaces”, Ann. of Math. (2) 139:1 (1994), 183-225. MR Zbl

[Getzler 1998] E. Getzler, “Topological recursion relations in genus 2”, pp. 73-106 in Integrable systems and algebraic geometry (Kobe/Kyoto, 1997), edited by M.-H. Saito et al., World Sci. Publ., River Edge, NJ, 1998. MR Zbl

[Graber and Vakil 2005] T. Graber and R. Vakil, "Relative virtual localization and vanishing of tautological classes on moduli spaces of curves”, Duke Math. J. 130:1 (2005), 1-37. MR Zbl

[Looijenga 1995] E. Looijenga, "On the tautological ring of $M_{g}$ ", Invent. Math. 121:2 (1995), 411419. MR Zbl

[Petersen 2016] D. Petersen, "Tautological rings of spaces of pointed genus two curves of compact type", Compos. Math. 152:7 (2016), 1398-1420. MR Zbl

[Petersen and Tommasi 2014] D. Petersen and O. Tommasi, "The Gorenstein conjecture fails for the tautological ring of $\overline{\mathcal{M}}_{2, n}$ ", Invent. Math. 196:1 (2014), 139-161. MR Zbl

[Rulla 2001] W. F. Rulla, The birational geometry of moduli space M(3) and moduli space M(2,1), Ph.D. thesis, University of Texas at Austin, 2001, Available at http://search.proquest.com/docview/ 304719193. MR

[Rulla 2006] W. F. Rulla, "Effective cones of quotients of moduli spaces of stable $n$-pointed curves of genus zero", Trans. Amer. Math. Soc. 358:7 (2006), 3219-3237. MR Zbl

[Tarasca 2015] N. Tarasca, "Double total ramifications for curves of genus 2", Int. Math. Res. Not. 2015:19 (2015), 9569-9593. MR Zbl 
Communicated by David Eisenbud

Received 2015-11-09

dawei.chen@bc.edu

tarasca@math.utah.edu
Revised 2016-06-17 Accepted 2016-09-10

Department of Mathematics, Boston College, Maloney Hall, 140 Commonwealth Ave, Chestnut Hill, MA 02467-3806, United States

Department of Mathematics, University of Utah, 155 S 1400 E, Salt Lake City, UT 84112, United States 


\section{Algebra \& Number Theory}

msp.org/ant

\section{EDITORS}

MANAGING EDITOR

Bjorn Poonen

Massachusetts Institute of Technology

Cambridge, USA

\author{
EDITORIAL BOARD CHAIR \\ David Eisenbud \\ University of California \\ Berkeley, USA
}

BOARD OF EDITORS

$\begin{aligned} \text { Dave Benson } & \text { University of Aberdeen, Scotland } & \text { Susan Montgomery } & \text { University of Southern California, USA } \\ \text { Richard E. Borcherds } & \text { University of California, Berkeley, USA } & \text { Shigefumi Mori } & \text { RIMS, Kyoto University, Japan } \\ \text { John H. Coates } & \text { University of Cambridge, UK } & \text { Raman Parimala } & \text { Emory University, USA } \\ \text { J-L. Colliot-Thélène } & \text { CNRS, Université Paris-Sud, France } & \text { Jonathan Pila } & \text { University of Oxford, UK } \\ \text { Brian D. Conrad } & \text { Stanford University, USA } & \text { Anand Pillay } & \text { University of Notre Dame, USA } \\ \text { Hélène Esnault } & \text { Freie Universität Berlin, Germany } & \text { Victor Reiner } & \text { University of Minnesota, USA } \\ \text { Hubert Flenner } & \text { Ruhr-Universität, Germany } & \text { Peter Sarnak } & \text { Princeton University, USA } \\ \text { Sergey Fomin } & \text { University of Michigan, USA } & \text { Joseph H. Silverman } & \text { Brown University, USA } \\ \text { Edward Frenkel } & \text { University of California, Berkeley, USA } & \text { Michael Singer } & \text { North Carolina State University, USA } \\ \text { Andrew Granville } & \text { Université de Montréal, Canada } & \text { Vasudevan Srinivas } & \text { Tata Inst. of Fund. Research, India } \\ \text { Joseph Gubeladze } & \text { San Francisco State University, USA } & \text { J. Toby Stafford } & \text { University of Michigan, USA } \\ \text { Roger Heath-Brown } & \text { Oxford University, UK } & \text { Ravi Vakil } & \text { Stanford University, USA } \\ \text { Craig Huneke } & \text { University of Virginia, USA } & \text { Michel van den Bergh } & \text { Hasselt University, Belgium } \\ \text { Kiran S. Kedlaya } & \text { Univ. of California, San Diego, USA } & \text { Marie-France Vignéras } & \text { Université Paris VII, France } \\ \text { János Kollár } & \text { Princeton University, USA } & \text { Kei-Ichi Watanabe } & \text { Nihon University, Japan } \\ \text { Yuri Manin } & \text { Northwestern University, USA } & \text { Efim Zelmanov } & \text { University of California, San Diego, USA } \\ \text { Philippe Michel } & \text { École Polytechnique Fédérale de Lausanne } & \text { Shou-Wu Zhang } & \text { Princeton University, USA }\end{aligned}$

PRODUCTION

production@msp.org

Silvio Levy, Scientific Editor

See inside back cover or msp.org/ant for submission instructions.

The subscription price for 2016 is US $\$ 290$ /year for the electronic version, and $\$ 485 /$ year (+\$55, if shipping outside the US) for print and electronic. Subscriptions, requests for back issues and changes of subscribers address should be sent to MSP.

Algebra \& Number Theory (ISSN 1944-7833 electronic, 1937-0652 printed) at Mathematical Sciences Publishers, 798 Evans Hall \#3840, c/o University of California, Berkeley, CA 94720-3840 is published continuously online. Periodical rate postage paid at Berkeley, CA 94704, and additional mailing offices.

ANT peer review and production are managed by EditFLow ${ }^{\circledR}$ from MSP.

\section{PUBLISHED BY}

- mathematical sciences publishers

nonprofit scientific publishing

http://msp.org/

() 2016 Mathematical Sciences Publishers 


\section{Algebra \& Number Theory}

Volume $10 \quad$ No. $9 \quad 2016$

Torsion pour les variétés abéliennes de type I et II

MARC HINDRY and NICOLAS RATAZZI

Galois-generic points on Shimura varieties

ANNA CADORET and ARNO KRET

Extremality of loci of hyperelliptic curves with marked Weierstrass points

DAWEI CHEN and NiCOLA TARASCA

$\bar{\Re}_{15}$ is of general type

GREGOR BRUNS

A vanishing theorem for weight-one syzygies

LAWRENCE EIN, ROBERT LAZARSFELD and DAVID YANG

1983

Effective cones of cycles on blowups of projective space

IZZET COSKUN, JOHN LESIEUTRE and JOHN CHRISTIAN OTTEM

Cluster algebras and category $\mathrm{O}$ for representations of Borel subalgebras of quantum affine algebras

DAVID HERNANDEZ and BERNARD LECLERC 